\title{
Use of Metallic Resonant Sensor in Torque Measurement Transfer Standard
}

\author{
Intiang J, DeGollier E, Rakowski R T, Jones B E, Cheshmehdoost ${ }^{1}$ A. \\ Brunel University \\ ${ }^{1}$ (ForceSenSys Ltd., Brunel Commercialisation Office) \\ Kingston Lane, Uxbridge, UB8 3PH, UK
}

\begin{abstract}
This paper will describe progress in the development of miniature metallic resonant Triple Beam Tuning Fork (TBTF) sensors for torque measurement applications. For high accuracy any torque transducer using these sensors ought to have low sensitivity to parasitic influences such as bending moments and lateral forces, which can only be achieved with reduced sizes. Compared to the original $15.5 \mathrm{~mm}$ length vibrating tine, the lateral forces are reduced by $52 \%$ for a $9 \mathrm{~mm}$ tine length and by $80 \%$ for $6 \mathrm{~mm}$ tine. High stiffness of these metallic resonant sensors will additionally reduce the occurrence of wrong measurement values as a result of overload damage. To support the use of these sensors in industrial applications a transfer standard using this technology is recommended.
\end{abstract}

Key Words: Torque Measurement, Calibration, Resonant Sensor, Transfer Standard

\section{Introduction}

Quality of measurement in industry requires the measured value to be compared to reference standards, which are traceable to the base SI unit. This can be achieved by using transfer standards. The most common torque transfer standard, widely used in laboratories and in industry, is based on strain gauge sensors. Low sensor output signals require surface strain on the sensing elements to approach the elastic limit in order to obtain a measurable signal over a wide measuring range. As a result, the overload capability of strain gauge torque transducers is limited to about $20 \%$ of full range; this is too low for many applications. To overcome this problem, i.e. to increase overload capability whilst maintaining accuracy and resolution, work has been started to develop a new torque transfer standard using metallic resonant sensors [1]. Additionally, metallic resonant sensors give a frequency signal compatible with digital circuitry, so that analogue-to-digital conversion can be eliminated. Moreover, the resonant sensor provides better long-term stability, since the frequency signal is not dependent on the amplitude of the electrical signals and hence accuracy is not limited by signal-to-noise ratio.

In 2005, Yan et al [1] showed that the metallic resonant sensor based on TBTF design showed high overload capability in the measurement of torque. Full scale operation is achieved with less than 400 microstrain, which is about five times stiffer than for resistance strain gauge torque transducers. However, the TBTFs used in this research were $40 \mathrm{~mm}$ long (characteristics shown in Table 1). Finite Element Analysis (FEA) indicated that the TBTF not only measures the changes in tensile/compressive strain at a $45^{\circ}$ angle to the axis of the shaft caused by torque but also experiences some shear strain and twist that will affect the final accuracy of the torque transducer. This effect could be minimized if it was possible to reduce the overall size of the TBTFs, whilst maintaining the stiffness of the resonant sensors. Figure 3 shows the sensors spot-welded onto the outer shoulders of the torque transducer at $\pm 45^{\circ}$ relative to the neutral axis of the shaft (one on either side), i.e. the resonators are set perpendicular to each other.

\section{Design and Fabrication}

The combination of processes used in resonator fabrication, photo-etched TBTFs with drive and pick-up piezoelectric thick-film elements printed directly onto the device surface, presents low-cost manufacturing opportunities for mass batch production [1]. Figure 1a shows a plan view of the resonator structure with PZT drive and detection elements. The magnitude of the output of the lead zirconate titanate (PZT) element depends upon the piezoelectric properties of the deposited layer, its thickness and the stress or voltage applied. In trying to reduce the size of the sensor there is a danger that the piezoactuator might not be able to excite the stiffer tines, so PZT printing is a physical limitation in size reduction. Finite 
element analysis (FEA) was employed to simulate the modal behaviour and stress distribution of the resonator in order to optimise the positioning of the thick-film PZT elements on the structure.

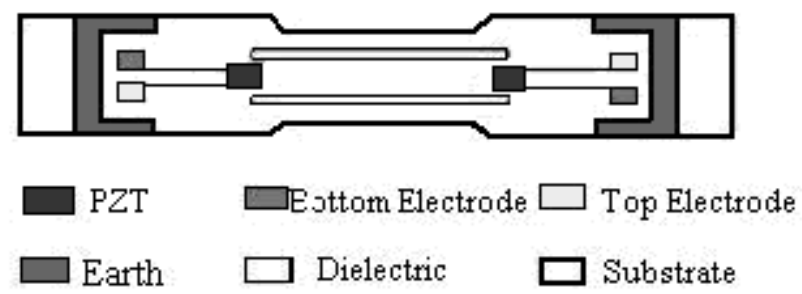

Figure 1a Plan view of the resonator structure with PZT drive and detection elements.

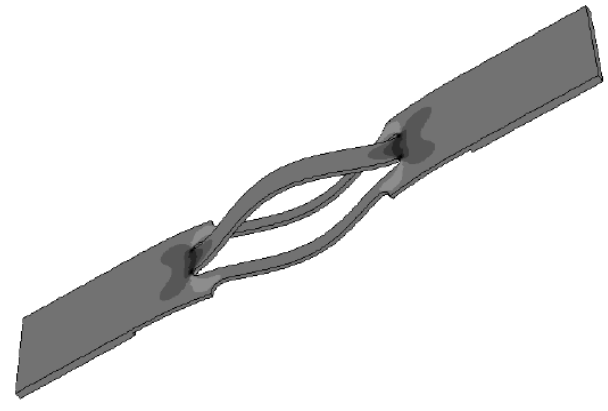

Figure 1b Stress contour plot for the resonator in mode 3 .

The triple beam tuning fork has a planer structure and is inherently more dynamically balanced when compared to a single beam or an out-of-plane double beam structure vibrating in flexural modes. Balanced structures dissipate less energy through their supports and therefore possess an intrinsically higher mechanical quality factor. The sensor is designed to oscillate in a third harmonic differential mode, where the central beam vibrates in anti-phase with the outer beams (mode 3) to minimise mechanical energy losses from the resonator. Figure $1 \mathrm{~b}$ shows the operational modal behaviour of the sensor modelled by FEA.

The first phase of the miniaturisation process was achieved by simply reducing the overall length by $50 \%$ from $40 \mathrm{~mm}(\mathrm{~T} 15,15.5 \mathrm{~mm}$ tine) to $20 \mathrm{~mm}$ (T9, 9mm tine) [2]. The latest version of the sensor $\mathrm{T} 6$ has reduced the size by $70 \%$ from the original, as shown in figure 2 . For the latest embodiment of the resonant sensor FEA analysis showed that a further reduction of $50 \%$ in the overall size was not possible due to the requirements of the PZT geometry and increased difficulty in exciting the short tine length.

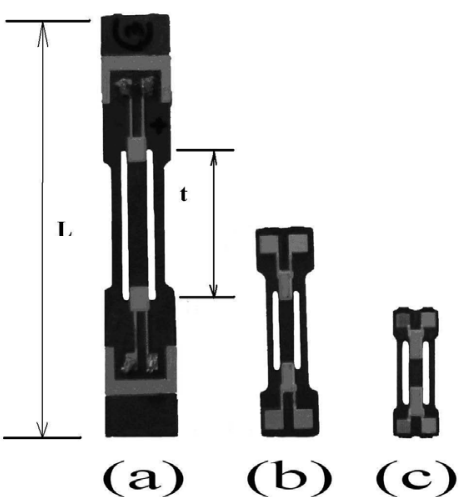

Figure 2 Miniaturisation of the metallic TBTF resonant sensors (a) T15 (b) T9 (c) T6

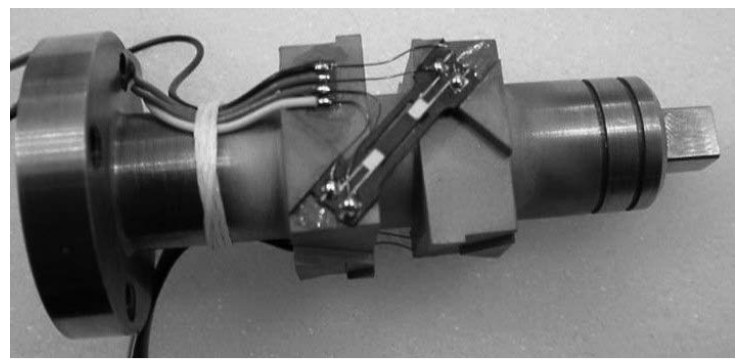

Figure 3 Torque transducer with metallic TBTF resonant sensor

The strategy adopted for the miniaturisation process was to maintain the tine cross-sectional area, roughly at $0.4 \mathrm{~mm}^{2}$, reduce the connector and mounting geometry but maintain the area of the PZT elements. Fundamental resonator operation and characteristics of the three miniaturized metallic TBTF resonant sensors are summarized in table 1. 
Table 1 Resonator operation and characteristics of the three miniaturized metallic TBTF resonant sensors ( ${ }^{*}$ Results previously published [2])

\begin{tabular}{|c|c|c|c|c|c|c|c|}
\hline SENSOR & Length L (mm) & Tine $\mathbf{t}(\mathbf{m m})$ & $\mathbf{f}_{\mathrm{o}}(\mathbf{k H z})$ & $\mathbf{Q}$ & Stability & Repeatability & $\begin{array}{c}\text { Temperature } \\
\text { Sensitivity } \\
\mathbf{H z} /{ }^{\circ} \mathbf{C}\end{array}$ \\
\hline T15 $^{\star}$ & 40 & 15.5 & 6 & 870 & $0.03 \%$ & $<0.01 \%$ & 0.75 \\
\hline T9 $^{\star}$ & 20 & 9 & 26 & 4002 & $0.04 \%$ & $0.02 \%$ & 2.5 \\
\hline T6 & 12 & 6 & 40 & 1600 & $0.02 \%$ & $<0.01 \%$ & 5.2 \\
\hline
\end{tabular}

\section{FEA Analysis of Torque Transducer}

\subsection{Introduction}

The transducer in figure 3 was modelled in FEA and the results were compared with actual test measurements. Operating at $5 \mathrm{Nm}$, a 100 microstrain reading compares with a level of 1800 microstrain usually required for torque transducers based on resistance strain gauges, this will give a major advantage in application where high overload capability is required

\subsection{Parasitic Influences}

When torque is applied, it is expected that there will be a small amount of twist in the TBTF sensor when the shaft strain is in shear, creating different amounts of tension in the outer tines and the middle tine. These differences would have an effect on the accuracy of any torque reading. When the sensor is loaded with a one-directional force, as in a load cell, the sensor shows a small variation across three tines [3] as shown in figure 4a. Because the angle actually changes from 45 degrees as the material strains, the sensor will have several different forces acting on it. There will be tensile strain, which is the quantity being measured but there also will be a shear strain, as the once co-linear sides of the mounting surfaces move away from each other. The structure will also undergo a small degree of twist, disproportionally raising the strain in the side tines in relation to the central tine. The resulting distortions in the sensor in torque measurement can be seen in figure $4 \mathrm{~b}$, amplified 1000 times. While the shear is quite obvious, it can be seen that there is also some twist in the structure. This effect could be minimized if it was possible to reduce the overall size of the metallic TBTF resonant sensor, whilst maintaining the stiffness of the resonant sensors.

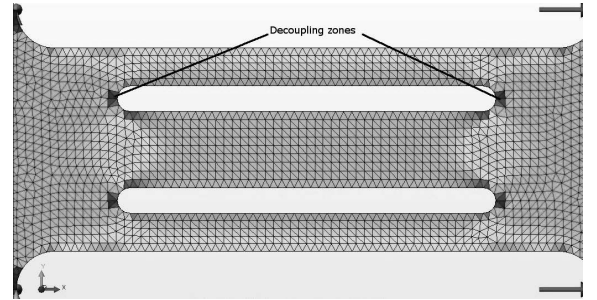

Figure 4a TBTF with 1 directional force along its axis (load cell configuration)

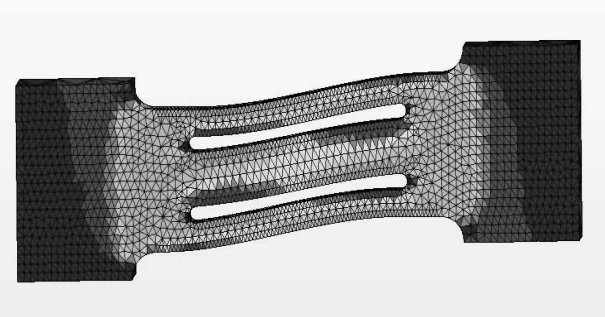

Figure $4 \mathbf{b}$ TBTF with distortion configuration

\subsection{Stress Distribution Averaging across Resonant Tines}

Figure 5a shows an example FEA result for the three metallic TBTF resonant sensors T15, T9, T6 in the same condition of mounting and loading with a $20 \mathrm{~N}$ shear force at one end. The average difference in stress distribution between the 3 tines compared to the T15 sensor is a reduction of $52 \%$ for the T9 sensor and reduced by $80 \%$ for T6 sensor. 


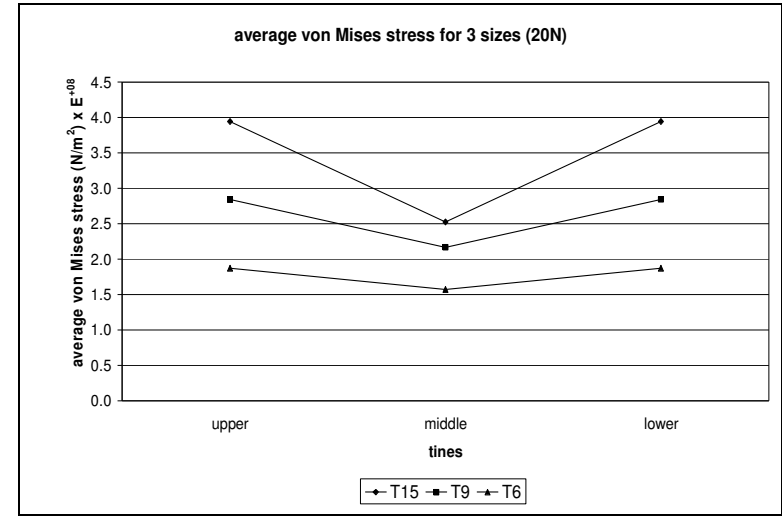

Figure 5a FEA results of the average von Mises stress on three tines for the T15, T9, and T6 sensors with an applied 20N shear force

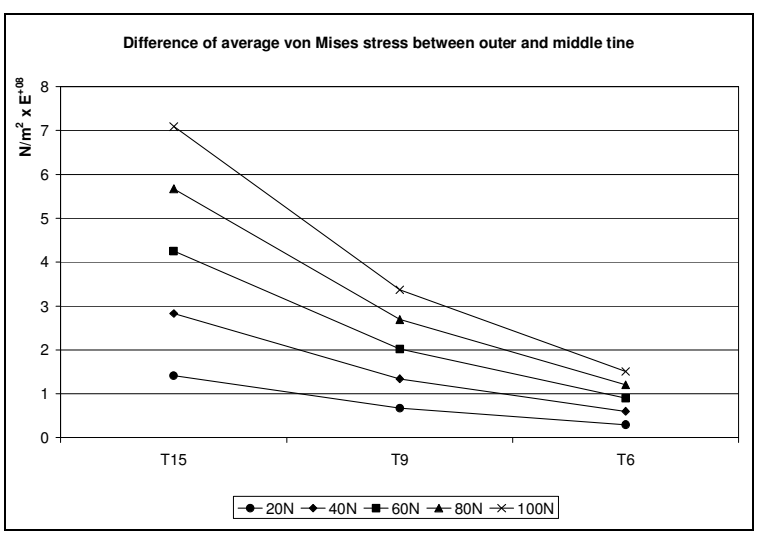

Figure $5 b$ FEA results of the difference of the average von Mises stress between the outer and middle tines for the T15, T9, and T6 sensors for applied $20-100 \mathrm{~N}$ shear force

Figure $5 \mathrm{~b}$ illustrates a further FEA analysis on the three resonant sensors for applied shear loads from $20 \mathrm{~N}$ to $100 \mathrm{~N}$. It is clearly seen that when the size of the sensor is reduced, the difference in the average stress distribution on three vibrating tines is decreased, concluding that reducing the overall size can satisfy the requirement to improve the accuracy of the torque transducer with the metallic TBTF resonant sensors.

\section{Characteristics of T6 Resonant Sensor}

Date: 11/06/08 Time: 13:07

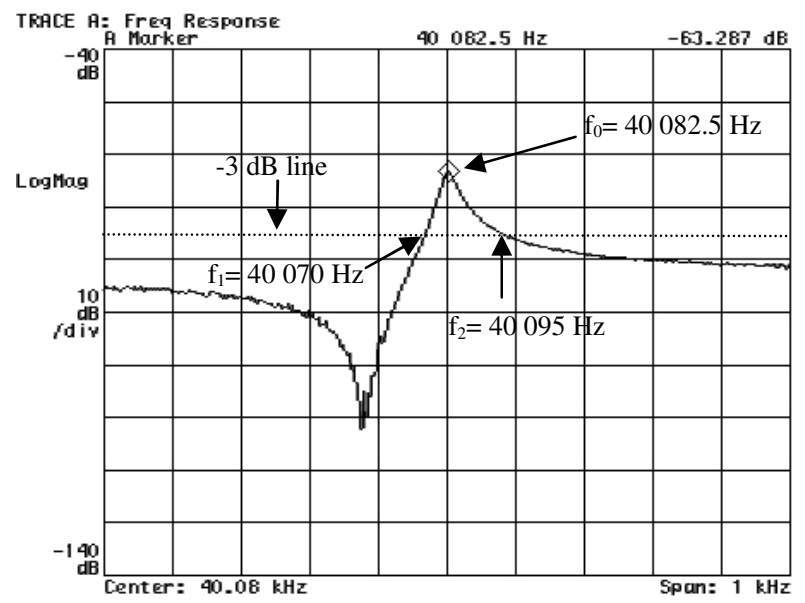

Figure 6 Resonant frequency signal of T6 sensor
Figure 6 illustrates the amplitude-frequency response from the first manufactured batch of T6 sensors and shows the third mode of vibration characteristic. From the result the value for the centre frequency is about $40 \mathrm{kHz}$ and the quality factor (Q) obtained from this characteristic is calculated to be about 1600 .

For the repeatability measurement of T6 sensor, the default frequency resolution was $2.5 \mathrm{~Hz}$, the standard deviation from the mean value of the resonant frequencies was found to be $\pm 1.6 \mathrm{~Hz}$. The standard deviation for the stability test was found to be $\pm 5.2 \mathrm{~Hz}$. Finally, the temperature coefficient of the resonator was measured at $5.2 \mathrm{~Hz} /{ }^{\circ} \mathrm{C}$.

\section{Overload Capability}

Strain gauge transducers produce low amplitude output signals, thus the surface strain is generally designed to approach the elastic limit of the sensing element in order to obtain a measurable value. As a result, the overload capability of the strain-gauge-based torque transducer will be limited by this factor [1]. The overall accuracy of the torque measuring system is determined by mechanical disturbance variables and the uncompensated thermal and humidity effects. In addition any overloading or damage to the torque transducer is a significant factor in obtaining the wrong measurement values [5] during testing or use in industrial applications. Such errors in measurement values might not be detected until the next calibration event. From the torque measurement point of view, the overload is the quantity that is actually 
of interest in normal operation, as it is usually an indicator of a developing fault or failure in a manufacturing application. It is additionally very hard to predict the level or nature of such a overload fault. In most cases the manufacturing system will have the appropriate overload protection to prevent damage to the plant and possibly the transducer, such as switching off the drive part when the overload is built up. However, such mechanical overload protection devices respond with a certain time delay, as the occurrence of the overload peaks is highly dynamic [4], so it is not enough to simply protect the transducer with these protective measure and hope that no damage occurs.

This problem with overload capability is one of the factors which could lead to the development of the new torque transfer standard using sensors with high stiffness. Evidence shows that the TBTF resonant sensor, fabricated with stiff metallic structures will provide better overload capability than strain gauges, at the same time as increasing the torque measuring range [1].

\section{Transfer Standard}

In metrology, traceability to the base $\mathrm{SI}$ unit is very important to ensure that the measured value is accurate. From the definition of torque, which is the product of force and distance, it can be traceable to the fundamental units of mass, length and time. The hierarchy of metrology is as follow; primary standard, secondary standard, calibration machine and measuring instrument.

In torque calibration, the measured quantity (torque) is disseminated or transferred from the National Standard to a reference standard, then to working standards and finally to test devices for applications by using torque transfer standard. Importantly, the transfer transducer should not have incorrect measurement properties. The damage to the transducer by overloading, which results in an error in the measurement value, can not be detected from an incorrect zero signal but only during recalibration [5].

The T9 TBTF torque transducer was evaluated at the National Physical Laboratory (NPL) in UK against the Torque Standard Machine (Deadweight Lever-beam). Calibration was carried out to British Standard (BS) 7882. Parameters measured included repeatability, reproducibility, linearity, residual deflection, reversibility and uncertainty. Moreover, stability and creep test had also been investigated to obtain more information on the transducer.

A series of ten readings were taken at each calibration point to minimise the influence of any fluctuations in the reading. A one-minute waiting time enabled the system to stabilise after each change of torque increment. The calibration result is shown in Table 2.

Table 2 Calibration result of torque transducer according to BS7882

\begin{tabular}{|c|c|c|c|c|c|}
\hline $\begin{array}{c}\text { Torque } \\
\text { (N.m) }\end{array}$ & $\begin{array}{c}\text { Relative error of } \\
\text { repeatability } \mathbf{R}_{\mathbf{1}}\end{array}$ & $\begin{array}{c}\text { Relative error of } \\
\text { reproducibility } \mathbf{R}_{\mathbf{2}}\end{array}$ & $\begin{array}{c}\text { Relative error of } \\
\text { interpolation } \mathbf{E}_{\mathrm{it}}\end{array}$ & $\begin{array}{c}\text { Relative error } \\
\text { of zero } \mathrm{E}_{\mathrm{it}}\end{array}$ & $\begin{array}{c}\text { Relative error of } \\
\text { reversibility } \mathbf{E}_{\mathrm{h}}\end{array}$ \\
\hline $\mathbf{0}$ & & & & $0.15 \%$ & \\
\hline $\mathbf{5}$ & $0.34 \%$ & $0.76 \%$ & $0.31 \%$ & & $-0.28 \%$ \\
\hline $\mathbf{1 0}$ & $0.13 \%$ & $0.99 \%$ & $-0.05 \%$ & & $-0.62 \%$ \\
\hline $\mathbf{1 5}$ & $0.15 \%$ & $0.80 \%$ & $-0.22 \%$ & & $-0.44 \%$ \\
\hline $\mathbf{2 0}$ & $0.00 \%$ & $0.39 \%$ & $-0.02 \%$ & & $-0.11 \%$ \\
\hline $\mathbf{3 0}$ & $0.00 \%$ & $0.15 \%$ & $-0.03 \%$ & & \\
\hline
\end{tabular}

The uncertainty of calibration at $95 \%$ confidence level $(k=2)$ is about $0.002 \%$.

Table 3 is the classification result of the torque transducer with $9 \mathrm{~mm}$ metallic TBTF resonant sensor. It shows that this torque transducer meets the requirements for a class 1 transfer standard.

Table 3 The classification result of torque transducer with metallic TBTF resonator

\begin{tabular}{|c|c|c|c|c|c|c|}
\hline $\begin{array}{c}\text { Relative error of } \\
\text { repeatability } \mathbf{R}_{\mathbf{1}}\end{array}$ & $\begin{array}{c}\text { Relative error of } \\
\text { reproducibility } \mathbf{R}_{\mathbf{2}}\end{array}$ & $\begin{array}{c}\text { Relative error of } \\
\text { interpolation } \mathrm{E}_{\mathrm{it}}\end{array}$ & $\begin{array}{c}\text { Relative error of } \\
\text { zero } \mathrm{E}_{\mathbf{z}}\end{array}$ & $\begin{array}{c}\text { Relative error of } \\
\text { reversibility } \mathrm{E}_{\mathrm{h}}\end{array}$ & Uncertainty & $\mathbf{a}$ \\
\hline 1 & 1 & 1 & 1 & 0.5 & 0.05 & 1 \\
\hline
\end{tabular}


From the calibration result, it is found that the class of accuracy of the torque transducer is limited by the resolution of the system display/indicator, shown as column ' $a$ ' in Table 3, where 'a' is a coeffficient which determines the lower limit of calibration when multiplied by the indicator resolution. Incorporating a display/indicating device with higher resolution would give aditional benefit in improving the accuracy of this torque transducer, possibly to the 0.5 or even 0.2 classification.

\section{Conclusion}

The metallic TBTF resonant sensor gives a direct frequency signal output, which has an advantage over conventional resistive strain gauges. The frequency signal is compatible with digital circuitry, so the analogue-to-digital conversion unit needed for strain gauges can be eliminated for this kind of torque transducer. Using metallic TBTF resonant sensors in a torque transfer standard is more convenient as the cost of the torque measurement system will be reduced compared to strain gauges. In addition, the resolution achievable using metallic resonant sensors would be as good as strain gauges, as frequency can be measured with quite high accuracy, which is very important for transfer standards. Moreover, resonant sensors provide better long-term stability since the frequency signal is not dependent on the amplitude of the electrical signals and the measurement accuracy is not limited by the signal-to-noise ratio, as occurs with an analogue signal.

The most common torque transfer standard is based on strain gauge sensors, where the overload capability is too low for many applications. A new torque transfer standard using metallic TBTF resonant sensors is proposed to overcome this problem, whilst maintaining accuracy and resolution. Measurement errors due to lateral forces, which would degrade the accuracy of such a torque transducer, were reduced by $80 \%$ when using the latest embodiment of the TBTF sensor. The new T6 sensor design, whose overall size has been reduced by $70 \%$, has maintained the high stiffness of the original T15 metallic resonant sensor. This paper shows the first test results for the T6 sensor, whose overall length is only $12 \mathrm{~mm}$. The stiffness of these metallic structures will reduce the occurrence of wrong measurement values due to any overload damage, which might occur in practical applications. Calibration results show that the a TBTF torque transducer incorporating the T9 sensors would be classified as a class 1 torque transfer standard. A torque transducer, incorporating the T6 sensors, is currently under construction and will be evaluated against national standards.

\section{References}

[1] Yan T, Jones B E, Rakowski R T, Tudor M J, Beeby S P and White N M. (2005) Stiff torque transducer with high overload capability and direct frequency output, IMEKO $19^{\text {th }}$ Int. Conf. on Force, Mass, Torque and Density Measurements, February, Cairo.

[2] Intiang J, Weidenmuller J, Rakowski R T, Jones B E, Cheshmehdoost A. (2007) Characteristics of $9 \mathrm{~mm}$ Metallic Triple-Beam Tuning Fork Resonant Sensor. Sensors and their Applications XIV, Ed. S J Prosser and A Al-shamma'a: Institute of Physics Publishing.

[3] Eric D. (2007) Design of a retrofit power-sensing coupling for pumping systems using triple-beam tuning fork strain gauges. Dissertation MSc Engineering Design: School of Engineering and Desing, Brunel University.

[4] Rainer, S. and Georg, W. (2002) Measuring Torque Correctly. Germany:HBM.

[5] Sven, K. (2008) High Precision Torque Measurement System. [www] Available from: http://www.hbm.com/fileadmin/mediapool/techarticles/2008/2008_kuhn-highprecision_torque_en.pdf [Accessed 5th Jan 2009] 